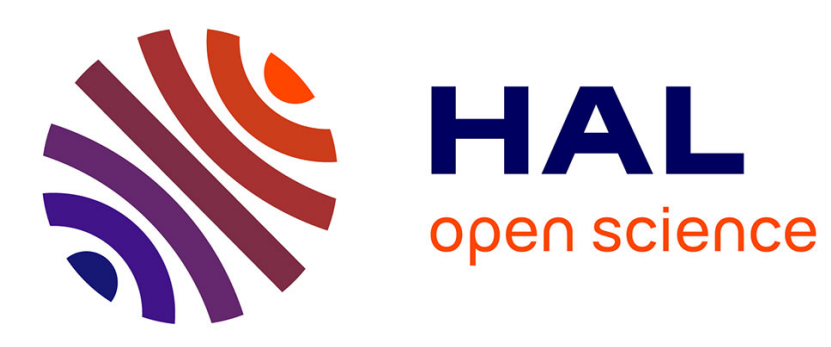

\title{
A multidimensional approach to the expression of phenotypic plasticity
}

Thibaut Morel-Journel, Virginie Thuillier, Frank Pennekamp, Estelle Laurent, Delphine Legrand, Alexis Chaine, Nicolas Schtickzelle

\section{- To cite this version:}

Thibaut Morel-Journel, Virginie Thuillier, Frank Pennekamp, Estelle Laurent, Delphine Legrand, et al. A multidimensional approach to the expression of phenotypic plasticity. Functional Ecology, 2020, 34 (11), pp.2338-2349. 10.1111/1365-2435.13667 . hal-03013284

\author{
HAL Id: hal-03013284 \\ https://hal.science/hal-03013284
}

Submitted on 26 Nov 2020

HAL is a multi-disciplinary open access archive for the deposit and dissemination of scientific research documents, whether they are published or not. The documents may come from teaching and research institutions in France or abroad, or from public or private research centers.
L'archive ouverte pluridisciplinaire HAL, est destinée au dépôt et à la diffusion de documents scientifiques de niveau recherche, publiés ou non, émanant des établissements d'enseignement et de recherche français ou étrangers, des laboratoires publics ou privés. 
Title: A multidimensional approach to the expression of phenotypic plasticity

\section{Authors and affiliations:}

Thibaut Morel-Journel ${ }^{1}$, Virginie Thuillier ${ }^{1}$, Frank Pennekamp ${ }^{1,2}$, Estelle Laurent ${ }^{1}$, Delphine Legrand ${ }^{1,3}$, Alexis S. Chaine $e^{3,4}$, Nicolas Schtickzelle ${ }^{1}$

${ }^{1}$ Université catholique de Louvain, Earth and Life Institute, Biodiversity Research Centre, Croix du Sud 4, L7.07.04, 1348 Louvain-Ia-Neuve, Belgium

2 University of Zurich, Department of Evolutionary Biology and Environmental Studies, Winterthurerstrasse 190, 8057 Zurich, Switzerland

${ }^{3}$ Station d'Ecologie Théorique et Expérimentale du CNRS UMR5321, 2 route du CNRS, 09200 Moulis, France

${ }^{4}$ Institute for Advanced Studies in Toulouse, Toulouse School of Economics, 21 allée de Brienne, 31015 Toulouse, France

\section{Corresponding author:}

Thibaut Morel-Journel

BIOEPAR, INRAE, Oniris, 44307, Nantes, France

thibaut.morel-journel@inrae.fr

\section{Acknowledgments}

This study was supported by the F.R.S.-FNRS and UCL (ARC 10-15/031). TMJ was supported by a Move-In-Louvain Marie Curie Action postdoctoral fellowship; VT was supported by a PhD grant from UCLouvain (ARC 10-15/31) and by a FRIA PhD grant. FP was supported by a PhD grant from UCLouvain-FSR. EL is teaching assistant at UCLouvain. DL is a previous postdoctoral researcher with NS funded by the F.R.S.-FNRS and UCLouvain-FSR, who is now at the SETE and supported by the 
Agence Nationale de la Recherche through the POLLUCLIM project (ANR-19-CE02-0021-01). DL and ASC are a part of the Laboratoire d'Excellence (LABEX) entitled TULIP (ANR-10-LABX-41) held by SETE and ASC is part of IAST, which is supported by ANR grant ANR-17-EUR-0010 (Investissements d'Avenir program). NS is Senior Research Associate of the F.R.S.-FNRS. This work is contribution BRCXXX of the Biodiversity Research Centre at UCLouvain. We thank Dr. Morrissey, an anonymous reviewer and an anonymous editor for constructive comments on an earlier draft of this manuscript.

\section{Author Contributions}

ASC, VT and NS conceived and designed research questions and the methodology for the experiment. VT, FP and EL collected the data, using the digital image analysis workflow developed by FP and NS. TMJ, VT and NS analysed the data with contributions from DL and ASC. TMJ led the writing on the manuscript, with substantial contributions from ASC, DL and NS. All authors contributed critically to the drafts and gave final approval for publication.

\section{Data Availability Statement}

Should the manuscript be accepted, the experimental data supporting the results will be archived in Dryad and the data DOI will be included at the end of the article. 


\section{Abstract}

Phenotypic plasticity is increasingly recognized as a key element of eco-evolutionary dynamics, but it remains challenging to assess because of its multidimensional nature. Indeed, organisms live in complex environments where numerous factors can impact the phenotypic expression of traits (inter-environment axis), possess multiple traits that can influence each other's expression (intertrait axis), and differ in their genetic background (inter-genotype axis), which can not only impact the traits' values but also their plasticity. We designed a microcosm experiment using the protist Tetrahymena thermophila aimed at encompassing all these aspects of phenotypic plasticity. We exposed 15 distinct genotypes to 25 combinations of temperature and nutrient availability and assessed the plasticity of five phenotypic traits. Hence, we addressed six questions related to phenotypic plasticity: (1) do different environmental gradients show similar effects on a given trait? (2) Are the effects of two environmental gradients on a trait additive? (3) Do different traits show similar plastic response to a given environmental gradient? (4) Do the (co)variances between traits vary across environmental gradients? (5) Do genotypes differ in their plastic response to a given environmental gradient? (6) Are some genotypes more plastic than others across all traits? Our results show strong differences in the plastic response depending on the environmental gradient, not only regarding the shape of the reaction norm of the different traits tested, but also in the overall plasticity of the organisms. We did not find any covariance between traits that was consistent across all environments. Overall, our results suggest independent impacts of the environmental dimension considered on the observed plastic response. These results underline potential difficulties in generalising findings about plasticity to all environments and all traits. 
67 controlled microcosms, G-matrix, genotype x environment experiment, phenotypic plasticity,

68 phenotypic syndrome, Tetrahymena thermophila, variance partitioning

69 


\section{Introduction}

Phenotypic plasticity is increasingly recognized as a critical factor in mediating eco-evolutionary processes (Carroll, Hendry, Reznick, \& Fox, 2007; DeWitt \& Scheiner, 2004; Forsman, 2015; Hendry, 2015; Pigliucci, 2001). Research over the past decades has revealed the ubiquity of plasticity (Miner, Sultan, Morgan, Padilla, \& Relyea, 2005; Palacio-López, Beckage, Scheiner, \& Molofsky, 2015; Pigliucci, 2001; Price, Qvarnström, \& Irwin, 2003; West-Eberhard, 2003) and identified the developmental (Emlen \& Nijhout, 2000; Hoverman \& Relyea, 2007; Murren et al., 2015), genetic (Callahan, Dhanoolal, \& Ungerer, 2005; DeWitt \& Scheiner, 2004; Van Kleunen \& Fischer, 2005), and epigenetic (Auge, Leverett, Edwards, \& Donohue, 2017; Ledón-Rettig, Richards, \& Martin, 2012; Smith \& Ritchie, 2013) processes underlying plasticity. Plasticity occurs when a given genotype produces different phenotypes in response to external factors, which may increase its short-term success when confronted with varying environments (DeWitt \& Scheiner, 2004; Pigliucci, 2001). The value of such plasticity ranges from buffering the impacts of changing environmental conditions (Ghalambor, McKay, Carroll, \& Reznick, 2007; Reed, Waples, Schindler, Hard, \& Kinnison, 2010) to facilitating colonization of new habitats (Geng et al., 2016; Richards, Bossdorf, Muth, Gurevitch, \& Pigliucci, 2006; Yeh \& Price, 2004), and helping to cross "adaptive valleys" during population divergence and speciation (Agrawal, 2001; Pfennig et al., 2010; van Snick Gray \& Stauffer, 2004).

Much of the early research on phenotypic plasticity has focused on the response of traits to a single environmental gradient (e.g. Bruno \& Edmunds, 1997; Denver, Mirhadi, \& Phillips, 1998; Weider \& Pijanowska, 1993). Yet, natural environments are composed of a multitude of interacting factors potentially influencing the plastic expression of traits. For example, the growth rate of the seed beetle Callosobruchus maculatus varies with both temperature and the rearing host (Stillwell, Wallin, Hitchcock, \& Fox, 2007), and the laying date of great tit Parus major varies with both temperature and day-length (Gienapp, Väisänen, \& Brommer, 2010). However, our understanding of how multiple environmental gradients influence reaction norms remains limited (Westneat, Potts, Sasser, \& Shaffer, 2019). The effect of two environmental dimensions could be additive, antagonistic, 
synergistic or complementary. Determining how multiple environments influence plasticity is challenging because it requires measures of independent and joint effects of factors on the same genotype through fully factorial experimental designs.

Studies concerning multivariate plasticity focus on the interaction between multiple phenotypic traits and how each trait affects phenotypic plasticity in the other trait (Laughlin \& Messier, 2015; Westneat et al., 2019). Indeed, organisms are mosaics of traits interacting to influence fitness (Dochtermann \& Dingemanse, 2013; Legrand et al., 2016; Sih, Bell, \& Johnson, 2004), which raises several questions. Firstly, will a single environmental factor cause plastic changes on several traits, or will traits be independently influenced by different factors? Secondly, for a given environmental factor, will the change in each trait be in a similar direction and magnitude, and how will the covariances between traits be impacted? While considerable information has accumulated on the shapes of reaction norms for a single trait measured along a single environmental gradient, we still need to determine if reaction norms are generalizable across traits and/or environmental factors.

Moreover, genotypic variation in the shape of reaction norms further increases the complexity of understanding the multi-dimensional nature of phenotypic plasticity. For example, differences in reaction norms occur between populations of seed beetles from Burkina Faso and South India in their plastic response to temperature (Stillwell et al., 2007) and between populations of great tits from the Netherlands and the UK (Charmantier et al., 2008; Husby et al., 2010). To what extent reaction norms are similar across genotypes, similar across several traits of a given genotype, and whether some genotypes display more phenotypic plasticity on average across all traits than other genotypes is largely unexplored.

Phenotypic plasticity is therefore a complex phenomenon involving different kinds of multidimensionalities, usually addressed within separate studies. We identified three different axes along which this multidimensionality deserves deeper investigation (Fig. 1): (i) the inter-environment axis, concerning interactions between the response to multiple environmental factors (Westneat et al., 
123 the same environmental factors (Laughlin \& Messier, 2015), and (iii) the inter-genotype axis, concerning the relationship between the genetic background of organisms and their plastic response to environmental factors (Pigliucci, Murren, \& Schlichting, 2006; Richards et al., 2006). In this study, we investigated the complex interplay between these three axes in the expression of phenotypic plasticity. We used a series of controlled fully-factorial microcosm experiments in 15 clonal genotypes of a ciliated protist (Tetrahymena thermophila), where we manipulated temperature and nutrient concentration and measured the expression of five phenotypic traits linked to fitness, morphology and movement. These two environmental factors were chosen as they are both likely to vary over space and time in the natural habitat (freshwater bodies), where $T$. thermophila feeds on bacteria. Furthermore, with increasing temperature, metabolism accelerates leading to higher resource demand (Gerhard, 2019). This effect can be compensated by higher nutrient supply. The existence of phenotypic plasticity and its variation among genotypes have been shown previously in this species for various morphological, behavioural and fitness traits, but only along single environmental gradients, e.g., density-dependence of dispersal (Pennekamp, Mitchell, Chaine, \& Schtickzelle, 2014) and thermal performance (Jacob et al., 2018). Here, we examined plasticity in morphological, behavioural and fitness traits along two different environmental gradients, for each genetic line. We aimed at answering six questions (Fig. 1): (1) Do different environmental gradients show similar effects on a given trait? (2) Are the effects of two environmental gradients on a trait additive? (3) Do different traits show similar plastic environmental gradients? (5) Do genotypes differ in their plastic response to a given environmental 
We used 15 isogenic lines (referred to as "genotypes") of Tetrahymena thermophila, a free-living unicellular eukaryote (Supporting table 1). This species has been used for decades as a model species in molecular biology and physiology and more recently in evolutionary ecology (Chaine, Schtickzelle, Polard, Huet, \& Clobert, 2010; Fjerdingstad, Schtickzelle, Manhes, Gutierrez, \& Clobert, 2007; Jacob, isolated from natural populations across different sites in North America or created by subsequent crossings between genotypes in the laboratory (Supporting table 1). They were cultivated in lightcontrolled incubators (14h light/10h dark-cycle at $27^{\circ} \mathrm{C}$ ) in an axenic medium (PPYE $1 \mathrm{x}$ : $2 \%$ Proteose Peptone and $0.2 \%$ Yeast Extract [Becton Dickinson] diluted in ultrapure water [Altermatt et al., 2015]). Culture stocks were renewed every 10 days by inoculating a $2 \mathrm{ml}$ sample of fresh medium with $100 \mu \mathrm{l}$ of culture and maintained in $2 \mathrm{ml} 24$-well plates (CELLSTAR ref. 662160, Greiner BioOne, Belgium). All manipulations of axenic cultures were conducted under sterile conditions in a laminar flow hood (Ultrasafe $218 \mathrm{~S}$, Faster, Italy).

Experimental design

We performed a Genotype-by-Environment (G×E) experiment, where the Genotype factor was made up of the 15 clonal genotypes of $T$. thermophila. The Environment factor was all 25 possible combinations of five temperature $(\mathrm{T})$ levels $\left(15^{\circ} \mathrm{C}, 21^{\circ} \mathrm{C}, 27^{\circ} \mathrm{C}, 33^{\circ} \mathrm{C}\right.$ or $\left.39^{\circ} \mathrm{C}\right)$, and five nutrient concentration $(N)$ levels, as dilutions of the original PPYE $1 x$ medium $(0 x, 0.125 x, 0.25 x, 0.5 x$ or $1 x)$. The ranges of temperature and of nutrient concentration considered was the largest possible, given technical and biological constraints. Indeed, higher temperatures lead to massive mortality, while lower temperature and higher nutrient concentration would strongly hinder cell movement and metabolism. The complete experimental design then comprised $375 \mathrm{G} \times \mathrm{E} \times \mathrm{E}$ combinations (15 G x $5 \mathrm{~T}$ 
$171 \times 5 \mathrm{~N})$. The whole experiment was replicated three times leading to three fully independent

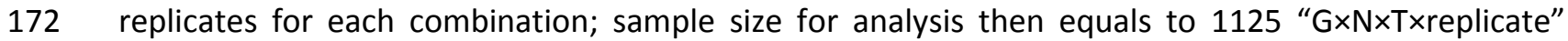
173 cultures.

Before each of the three repetitions of the experiment, one mother culture was initiated per genotype, by inoculating $5 \mathrm{ml}$ of PPYE $1 \mathrm{x}$ with $500 \mu$ l of monoculture of a given genotype and incubated in the standard culture conditions $\left(27^{\circ} \mathrm{C}\right.$, PPYE $\left.1 \mathrm{x}\right)$ for two days. Each of these 15 " $\mathrm{G}$ " mother cultures was then transferred in $100 \mathrm{ml}$ of fresh PPYE $1 \mathrm{x}$, conserved for five additional days in the same conditions to reach sufficient cell concentration for the experiment. Then, they were carefully homogenized and aliquoted into five smaller tubes, from which the old medium was removed by aspiration after five minutes of centrifugation at $250 \mathrm{~g}$ and replaced by $10 \mathrm{ml}$ of fresh PPYE nutrient, for each of the five different nutrient concentration levels. Finally, each of these 75 "G×N" culture was diluted to a standardized density of $300 \mathrm{kcell} / \mathrm{ml}$ with fresh PPYE medium at the same concentration, split into five $4 \mathrm{ml}$ cultures, each incubated at one of the five temperatures tested to start the experiment, giving 375 " $\mathrm{G} \times \mathrm{N} \times \mathrm{T}$ " cultures. Data were collected at two times during

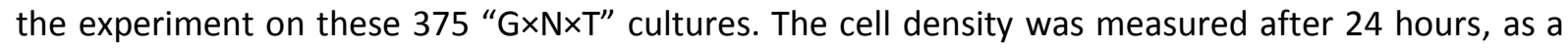
proxy for demography (population growth). Then, each culture was standardized at a density of 100 $\mathrm{kcell} / \mathrm{ml}$ through dilution into fresh medium and re-incubated with their respective nutrient concentration and temperature for one additional hour. The main reason to proceed in this way was to avoid a potential bias in the measure of movement behaviour arising when cell density is too high. In such conditions, "collisions" between swimming cells blur cell identity during tracking, cutting each movement trajectory into shorter and straighter bits, biasing speed and linearity. On the contrary, morphology is not largely affected over such a short time (1h) given that temperature and nutrient concentration conditions were unchanged.

Five metrics related to morphology, behaviour and fitness were quantified during the experiment using our standardized digital picture workflow (Pennekamp et al., 2014; Pennekamp, Schtickzelle, \& Petchey, 2015; Pennekamp \& Schtickzelle, 2013): density, cell size, cell shape, 
movement speed and movement linearity. For each culture, five $10 \mu \mathrm{l}$ samples were extracted after culture homogenization and each loaded into a chamber of a counting slide (Precision cell, Kima, Italy). The first three variables were obtained by analysing the pictures taken of each chamber under a dark field microscope: the density was estimated from the number of cells counted after 24 hours, the cell size as the area of the cross section in $\mu \mathrm{m}^{2}$, and the cell shape as the aspect ratio (i.e., major/minor axis ratio of a fitted ellipse; the minimal shape value was 1 for a perfectly round cell). The last two variables concerning movement were obtained from one video taken for a randomly chosen chamber among the five available for each culture. The videos were analysed using the BEMOVI R package (Pennekamp et al., 2015; http://www.bemovi.info) to reconstruct movement trajectories and obtain movement speed and linearity; linearity was quantified as the net-to-gross distance ratio (net distance is the Euclidian distance between the starting and arrival points; gross distance is the effective length of the trajectory; the maximal linearity value of 1 then indicates a perfectly straight path).

\section{Analysis of the results}

The statistical analyses of the experimental results were all performed using the R software (R Core team, 2018). To answer questions $1,2,3$ and 5, the response variables (i.e., the five phenotypic traits) were analysed using an ANOVA for variance partitioning and a random regression-mixed model for describing the mean reaction norm. First, we used an ANOVA model to quantify how the variance in the trait values is explained by the temperature $(T)$, the nutrient concentration $(N)$, the genotype $(\mathrm{G})$ and their interactions noted $T \times N, G \times T, G \times N$ and $G \times T \times N$. To account for the difference in the number of levels of each environmental factor (5) and of genotypes (15), we performed the variance partitioning for every possible subset of 5 genotypes among the 15 . For each term, we estimated the respective effect size of each factor and their interaction using the $\eta^{2}$ metric (Fritz, Morris, \& Richler, 2012), and then we computed the average value of $\eta^{2}$ over all these subsets as metrics of trait variance. 
Reaction norms, i.e., average trait values over the two-dimensional space of temperature and nutrient concentration, were assessed using quadratic random regression-mixed model analyses (Morrissey and Liefting 2016) using the Ime4 package (Bates et al., 2014). For each trait, the mixed model took the following form:

$$
z_{i, G}=a+b_{1} T_{i}+b_{2} T_{i}^{2}+b_{3} N_{i}+b_{4} N_{i}^{2}+b_{5} T_{i} N_{i}+c+d_{1, G} T_{i}+d_{2, G} T_{i}^{2}+d_{3, G} N_{i}+d_{4, G} N_{i}^{2}+d_{5, G} T_{i} N_{i}+e_{i}
$$

with $z_{i, G}$ the value of the trait for the experimental replicate $i$ for the genotype $g$ exposed to a given temperature $T_{i}$ and a given nutrient concentration $N_{i}$. To limit the size of the covariance matrix to ensure estimation of random effects and convergence of the models, we limited the interactions to the linear terms $\left(T_{i}\right.$ and $\left.N_{i}\right)$. Indeed, the inability to include interaction terms in the random slopes model is why we partitioned variance using an ANOVA approach above. The coefficients $a, b_{1}, b_{2}, b_{3}$, $b_{4}$ and $b_{5}$ were used to estimate the reaction norm, while accounted for potential non-linear relationships between the trait values and the environment, as well as the interactions between the environmental gradients. $\mathrm{T}$ and $\mathrm{N}$ values were each centred and standardized prior to the analysis to ensure independence between linear and quadratic terms as well as comparability of their size effect despite being expressed in different units (Shielzeth, 2010).

To answer question 4, for every environmental condition (the $5 \times 5$ environmental combinations), a G-matrix was computed to describe the (co)variances between the values of each trait across all genotypes. Each G-matrix included the variance of each trait on its diagonal, and the covariances between the five traits off diagonal. Since G-matrices are symmetrical, the covariances in the upper and lower triangles were identical and each matrix included 15 unique values: 5 variances and 10 covariances. To account for differences in the ranges of the trait values (e.g., linearity is a ratio always between 0 and 1 while density is always a positive integer), a mean standardization was performed by dividing the raw values by the mean value for each environmental condition (Delahaie et al., 2017; Kirkpatrick, 2009). We computed the effective number of dimensions of each G-matrix $n_{D}$, which can theoretically vary between 1 (the G-matrix is singular) to the number of traits (the Gmatrix is full-rank; here 5). The value of $n_{D}$ decreases when fewer dimensions are enough to describe 
the full information content in the G-matrix; and this can happen because of existing covariation between traits (one trait explains variation in other traits ) and/or heterogeneity in the level of variance in the different traits (if one trait has a much higher variance than others, it alone explains a high proportion of the matrix information, hence decreasing $n_{D}$ ) (Kirkpatrick, 2009). To disentangle the relative importance of these two aspects in giving low $n_{D}$ values, we computed an extra measure $n_{D}{ }^{\prime}$, corresponding to $n_{D}$ obtained in a set of $25 \mathrm{G}$-matrices with variances identical to the observed ones but covariances forced to 0 ; this $n_{D}{ }^{\prime}$ value sets the maximum number of effective dimensions in the absence of covariance between traits but taking heterogeneous trait variances into account. Then, we computed the ratio $\left(\left(n_{D}-1\right)-\left(n_{D}{ }^{\prime}-1\right)\right) /\left(n_{D}{ }^{\prime}-1\right)$ to quantify the reduction in effective number of dimensions in the G-matrices due to the existence of covariances among traits. genotype across $\mathrm{T}$ and across $\mathrm{N}$ as a proxy for the magnitude of their plasticity. The genotypes were ranked according to these coefficients (from 1 for the least plastic to 15 for the most plastic), and these ranks were compared using Kruskall-Wallis tests to identify differences in the average plasticity of genotypes over the five measured traits.

\section{Results}

(1) Do different environmental gradients show similar effects on a given trait?

The variance partitioning based on an ANOVA model allowed us to assess the relative importance of temperature $(T)$ and nutrient concentration $(N)$ on the variance of each of the five traits, for the levels of $\mathrm{T}$ and $\mathrm{N}$ considered in the experiment (Fig. 2). The results show clear differences in the amount of variance in the traits that were affected by the two environmental gradients. For instance, the shape of cells was only strongly impacted by $\mathrm{T}$ but not by $\mathrm{N}$, while the size of the cells was only

272 strongly impacted by N but not by T. Density, a measure of demography (population growth) given 273 that initial densities were normalized, was greatly affected by both $\mathrm{T}$ and $\mathrm{N}$, whereas movement 274 traits (speed and linearity) were impacted only slightly by T and $\mathrm{N}$. 
276 (2) Are the effects of two environmental gradients on a trait additive?

277 The interaction between $\mathrm{T}$ and $\mathrm{N}$ was systematically low for every trait (Fig. 2), indicating that the 278 impacts of the two environmental gradients on the response were mostly additive. This is confirmed

279

(3) Do different traits show similar plastic responses to a given environmental gradient?

While some traits showed similar plastic responses to a given environmental gradient, neither manipulated environmental feature influenced all traits in the same way. Density, cell shape and movement were consistently influenced by T. Interestingly, reaction norms of these traits were all quadratic (Fig. 3) and all reached their inflection points (maximum density, maximally elongated shape and minimal movement linearity) for intermediate values of T. Density and cell size were consistently plastic to $\mathrm{N}$. They exhibited a strong reduction of trait values at the lowest concentration (0x) compared to the other ones.

(4) Do the (co)variances between traits vary across environmental gradients?

There were large differences between the variances of the different traits, for all the G-matrices (Fig. 4A, 4B). The variance in shape was systematically low for all environments, while variances in speed and in linearity were on average higher, and tended to increase with temperature (Fig 4A). The impact of nutrient concentration on the variances was not as straightforward (Fig 4B). Overall, the co-variances of the G-matrices were smaller in absolute values than the variances, and most of them changed sign depending on the environment considered. The effective number of dimensions of the G-matrices $n_{D}$ ranged between 1.53 and 3 ( $n_{D}=1.98$ on average), indicating that the G-matrices were far from being full rank (Fig 4C). However, this was also the case of the values of $n_{D}{ }^{\prime}$, which ranged between 1.62 and 3.25. This suggests that the low dimensionality of the G-matrices is more likely due 
to heterogeneity in the variances of the five traits rather than to strong covariances among traits in the G-matrices.

\section{(5) Do genotypes differ in their plastic response to a given environmental gradient?}

We found considerable differences in raw trait expression between genotypes ( $G$ in Fig. 2), with genotypes presenting consistently higher (or lower) trait values across the whole $\mathrm{T}, \mathrm{N}$ or $\mathrm{T} \times \mathrm{N}$ environmental space (Fig. 3). We also found a non-negligible variation among genotypes in their reaction norms to the two environmental factors ( $G \times T+G \times N+G \times T \times N$ in Fig. 2), which varies from $17.5 \%$ for size to $36.2 \%$ in movement speed. Response to temperature was more variable among genotypes than response to nutrient concentration ( $G \times T \geq G \times N$ in Fig. 2).

\section{(6) Are some genotypes more plastic than others across all traits?}

The rankings of the genotypes according to the coefficient of variation in their phenotype at each trait across each environmental gradient revealed substantial differences in the plasticity of the genotypes depending on the trait considered (Fig. 5). Furthermore, some genotypes were significantly more plastic on average across all traits than others in their response to $\mathrm{T}$ (black dots in Fig. 5 left column; Kruskall-Wallis $\chi_{d f=14}^{2}=27.388 ; p=0.018$ ) but not in their response to $N$ (black dots in Fig. 5 right column; Kruskall-Wallis $\chi_{d f=14}^{2}=19.442 ; p=0.149$ ). Interestingly, we did not find systematic differences in which genotypes were either highly plastic or highly canalized on average across all traits across the two environments. Genotypes that showed higher plasticity on average across all traits for temperature were not those who were the most plastic on average across all traits for nutrient concentration (lines connecting genotypes between left and right panel in Fig. 5).

\section{Discussion}

We experimentally quantified the plastic responses of five traits for 15 genotypes of $T$. thermophila along gradients of both temperature and nutrient concentration to answers six questions about 
correlational effects of phenotypic plasticity in systems with multiple environmental gradients. We showed that: (1) different environmental gradients had different effects on a given trait, (2) temperature and nutrient concentrations generated additive impacts on the plastically-induced phenotypes, (3) a number of traits showed similar reaction norms to a given environmental gradient, but neither gradient influenced all traits in the same way, (4) no strong covariance between traits was conserved across environmental gradients, (5) the genotypes differed in their plastic response to a given environmental gradient, and (6) the genotypes differed in their overall plasticity across all traits. Our experiment led us to explore different axes of multidimensionality of the phenotypic response: the inter-environment axis by testing all combinations of five values for both environmental gradients, the inter-trait axis by measuring five different phenotypic traits, and the inter-genotype axis by using fifteen genetically distinct genotypes.

\section{The inter-environment axis}

We investigated plastic responses of $T$. thermophila to variation in nutrient concentration and temperature to ask how different environments influence specific traits. Interestingly, only a single trait -final density, a measure of demographic population growth- responded extensively to both environmental gradients. Such a strong plastic response to multiple environmental factors could result from the response of organisms to a single cue, itself correlated with multiple environmental gradients (Westneat, Hatch, Wetzel, \& Ensminger, 2011; Westneat et al., 2019). For example, the chrysophyte Synura echinulate responds in the same way to variations in light intensity and in temperature (Němcová, Neustupa, Kvíderová, \& Řezáčová-Škaloudová, 2010), which are thought to both convey the same information about environmental stress triggering the plastic response. The plasticity in cell density observed in our experiment is unlikely to correspond to this case, given the differences in the shapes of the reaction norms to temperature and nutrient concentration. We observed two plastic responses of the same trait to different environmental gradients that likely influence the trait separately. This can notably occur for traits whose variation can be underpinned 
by multiple processes that can each be affected by the environment independently. For instance, the leaf mass per area (LMA) was found to be plastic to irradiance (Sack, Melcher, Liu, Middleton, \& Pardee, 2006), nutrient abundance (Wright et al., 2005) and water abundance (Chin \& Sillett, 2016), which actually all affect different aspects of the leaf structure, thereby changing the LMA value (Poorter, Niinemets, Poorter, Wright, \& Villar, 2009). Similarly, the processes underpinning the cell division of $T$. thermophila, a component of fitness, depend on multiple factors related to the internal state of the cell and external cues. Therefore, density should exhibit plastic responses to multiple environmental gradients.

Although the plastic response to multiple environmental factors is seldom assessed, some studies have shown how interactions between multiple environmental gradients can affect the expression of reaction norms (Groot et al., 2016; Relyea, 2004; Stillwell et al., 2007), sometimes in a population-specific manner (Burghardt, 2016; Ris, Allemand, Fouillet, \& Fleury, 2004; Stillwell et al., 2007). Here, the $T \times N$ interaction was systematically negligible in our experiment. Even the $G \times T \times N$ interaction remained comparatively low for every trait, ruling out the possibility that the absence of a $\mathrm{T} \times \mathrm{N}$ interaction was an artefact of opposite $\mathrm{T} \times \mathrm{N}$ effects among genotypes. Our results suggest that plasticity to temperature and nutrient concentration are additive, and therefore more easily predictable in regard to global environmental changes. However, the disparity of results across studies to date suggests that potential interactions between plastic responses to multiple environmental factors might depend on the trait, environmental factor and species considered. Further studies investigating the interaction between effects of multiple environments are necessary to see how general additivity of plastic responses to two or more environmental gradients is.

\section{The inter-trait axis}

Understanding the relationship between the response of multiple traits to environmental gradients can shed light on past and future evolutionary changes of biological functions and especially multidimensional plasticity (Laughlin \& Messier, 2015). Syndromes, i.e., sets of covarying life-history traits, 
are found across taxa, e.g., r-K strategies (Roff, 2002), pace-of-life (Réale et al., 2010), oogenesis-

380 flight syndrome (Zera \& Denno, 1997), pollination syndromes (Fenster, Armbruster, Wilson, Dudash,

\& Thomson, 2004) or dispersal syndromes (Legrand et al., 2016). Understanding these covariation patterns, their origins and how they are impacted by environmental conditions is critical to understand the evolvability of those functions but has rarely been examined for multiple plastic traits.

Despite some similarities in reaction norm shape to a given environmental gradient for different traits, the covariances among traits observed during the experiments were actually largely smaller than trait variances. Indeed, the values on the diagonal of the G-matrices were overall greater than off diagonal values. Thus, we did not find the dispersal syndrome, previously identified among T. thermophila (Fjerdingstad et al., 2007; Jacob, Chaine, Huet, Clobert, \& Legrand, 2019; Jacob, Laurent, Morel-Journel, \& Schtickzelle, 2019); however our experimental design was not suitable to search for such a syndrome, as there was no possibility for cells to disperse. However, the high variance of the traits could represent a strong potential for future evolution, especially for those traits linked to movement. The speed of individuals and the linearity of their trajectories differed greatly between genotypes, especially for the highest temperatures. More importantly, the covariances were inconsistent across environmental gradients. Such context-dependency of trait covariation can have important ecological and evolutionary consequences. For instance, the lability of dispersal syndromes can allow organisms to adjust their dispersal movements according to both landscape characteristics and predatory risk (Winandy et al., 2019). Our study suggests that such labile trait covariation could be common, but we still lack empirical data to confirm this statement (but see Jacob, Laurent, et al., 2019; Legrand et al., 2016). Overall, the consistency of syndromes should be carefully assessed rather than assumed, especially in the most stressful conditions. 
through its plasticity. The raw values of the traits considered showed substantial variation linked to the genotype, as already shown in past studies (Fjerdingstad et al., 2007; Pennekamp et al., 2014). especially for traits linked to movement. Inter-genotype variation in traits linked to movement was especially high, and the lowest for cell density, which is consistent with standard selection models suggesting that traits more tightly linked to fitness are expected to show lower genetic variation (Kingsolver, Diamond, Siepielski, \& Carlson, 2012; Palacio-López et al., 2015). The inter-genotypes differences in the plastic response underline the high potential for evolution of reaction norms among T. thermophila. Especially, the partitioning of variances showed substantial G×E interactions, with $\mathrm{G} \times \mathrm{T}$ being systematically greater than $\mathrm{G} \times \mathrm{N}$, meaning that genotypes exhibited different reaction norms, especially to temperature. Those differences might reflect the environmental gradients encountered across the species' range, which spans from the north-eastern to the southern USA (Doerder, 2019; Zufall, Dimond, \& Doerder, 2013). However, the latitudinal variation in the origin of the 15 genotypes used in this study is not big enough to allow formal testing for such an effect. Further studies linking the phylogeography of $T$. thermophila genotypes with the difference in their plastic response offer an opportunity to study the evolution of plasticity along natural environmental gradients. Inter-genotype differences in plasticity at the trait level can translate into plasticity differences at the organism level (Forsman, 2015). In our experiment, we showed differences in overall plasticity of all five traits to temperature. These results are consistent with previous identification of thermal generalists and specialists among T. thermophila (Jacob et al., 2018), and with the differences in temperature variability across the specie's range (Doerder, 2019; Zufall et al., 2013). However, we did not find the same pattern in overall plasticity to nutrient concentration. Indeed, we did not find genotypes that were highly plastic overall, or plasticity specialists relative to others. Moreover, there was no correlation between the overall plasticity to temperature and to nutrient concentration. In other words, the overall plasticity of one genotype to one environmental gradient did not predict its overall plasticity to another gradient, or even the existence of differences 
431 in overall plasticity between genotypes for that other gradient. Therefore, one should be cautious

432 when separating specialists from generalists using a given environmental factor, as these definitions 433 heavily depend on the environmental factor considered. 
435 This study is a first attempt at addressing the multidimensional nature of phenotypic plasticity (inter-

436 environmental, inter-trait and inter-genotype) in a single fully factorial experimental design. Our

437 results have important implications for the evolution of phenotypic plasticity in nature where

438 organisms are exposed to multiple environmental gradients simultaneously. We showed that

439 plasticity in each trait depends on the environmental gradient, so does the existence of generalists

440 and specialists, i.e., genotypes that differed in their plasticity across all traits. At the trait level, the

441 environmental effects on plasticity of a given trait are largely additive instead of having complex non-

442 additive effects. Despite plasticity across multiple traits, there was little covariance among trait

443 across environments, as would be expected if traits formed a stable life-history syndrome, most

444 often as a consequence of genetic constraints. Together, these results suggest that plasticity of traits

445 to different environments should evolve largely independently of other traits or each environmental

446 cue rather than showing complex correlational evolutionary responses. However, this also means

447 findings concerning plasticity of one trait to one environmental gradient are more difficult to

448 generalize to other traits or environments, even for the same organism. 
Inter-genotype axis

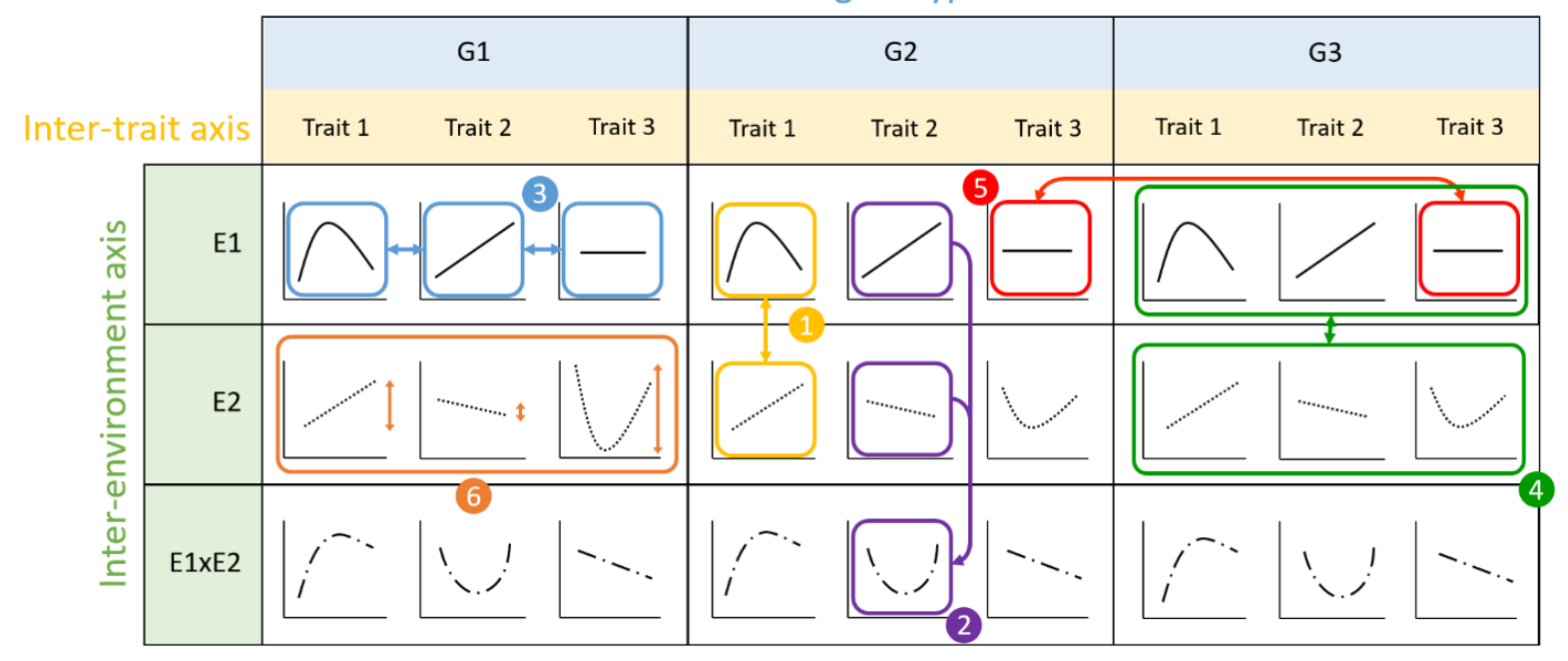

451

452 Figure 1: Schematic representation of the six different questions considered in this study and their

453 relationship to the three identified multidimensionality axes. We have represented hypothetical 454 reaction norms of three traits for three genotypes $(\mathrm{G} 1, \mathrm{G} 2, \mathrm{G} 3)$ in two environmental gradients (E1, 455 E2) and their interaction (E1×E2). We asked: (1) if different environmental gradients show similar 456 effects on the plasticity of a given trait, (2) if the effects of two environmental gradients on a trait are 457 additive, (3) if different traits show similar plastic response to a given environmental gradient, (4) if 458 (co)variances between traits vary across environmental gradients, (5) if there are differences 459 between genotypes in their plastic response to a given environmental gradient, and (6) if some 460 genotypes are more plastic than others across all traits. Each question is illustrated with a single example comparison only. 


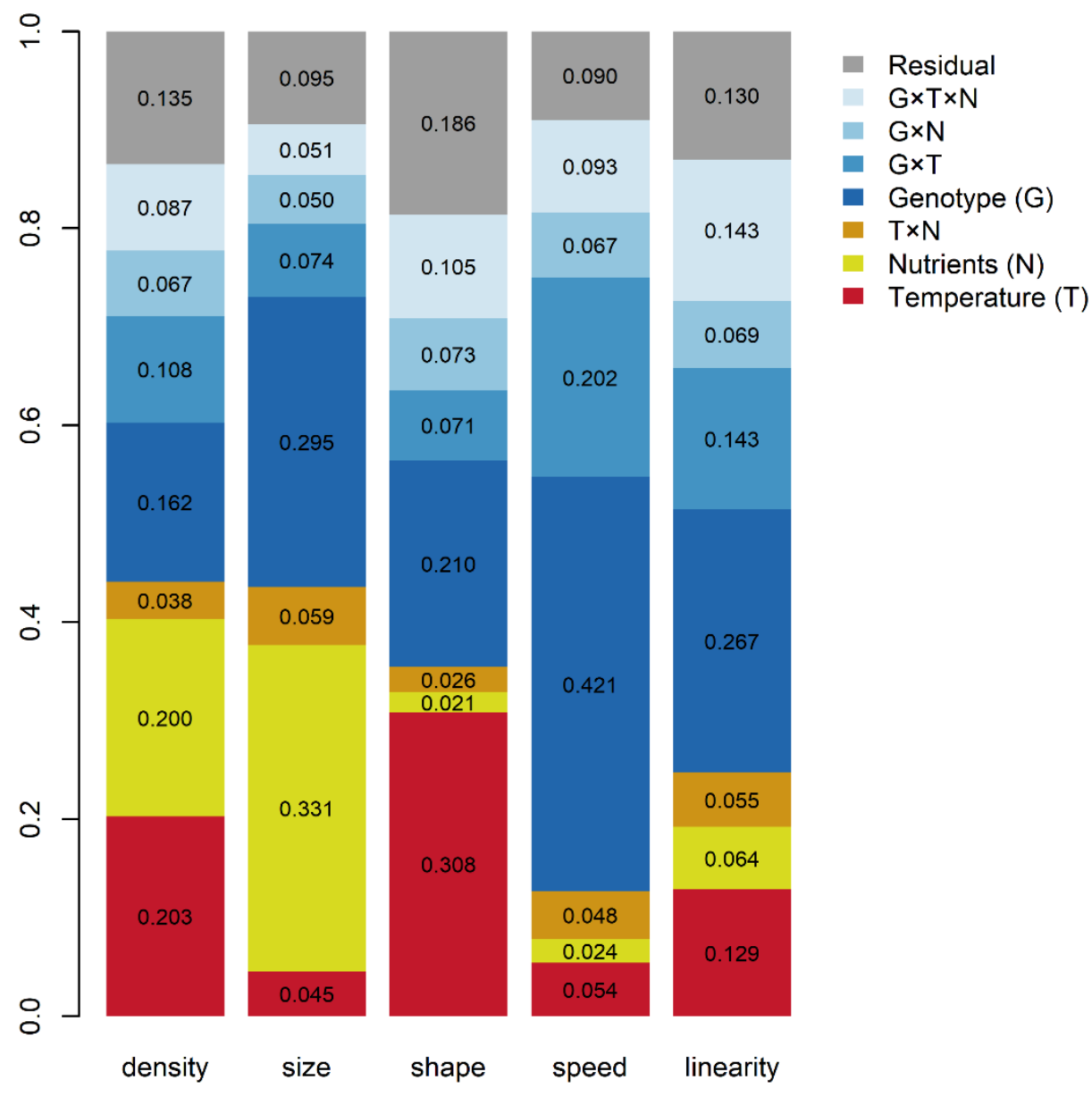

463

465 Figure 2: Proportion of variance $\left(\eta^{2}\right)$ explained by the different factors using an ANOVA model. To

466 accommodate differences in number of levels between temperature (5), nutrient concentration (5)

467 and genotypes (15), the ANOVA was fitted independently to all possible subsets of 5 genotypes and $468 \eta^{2}$ averages reported here. 

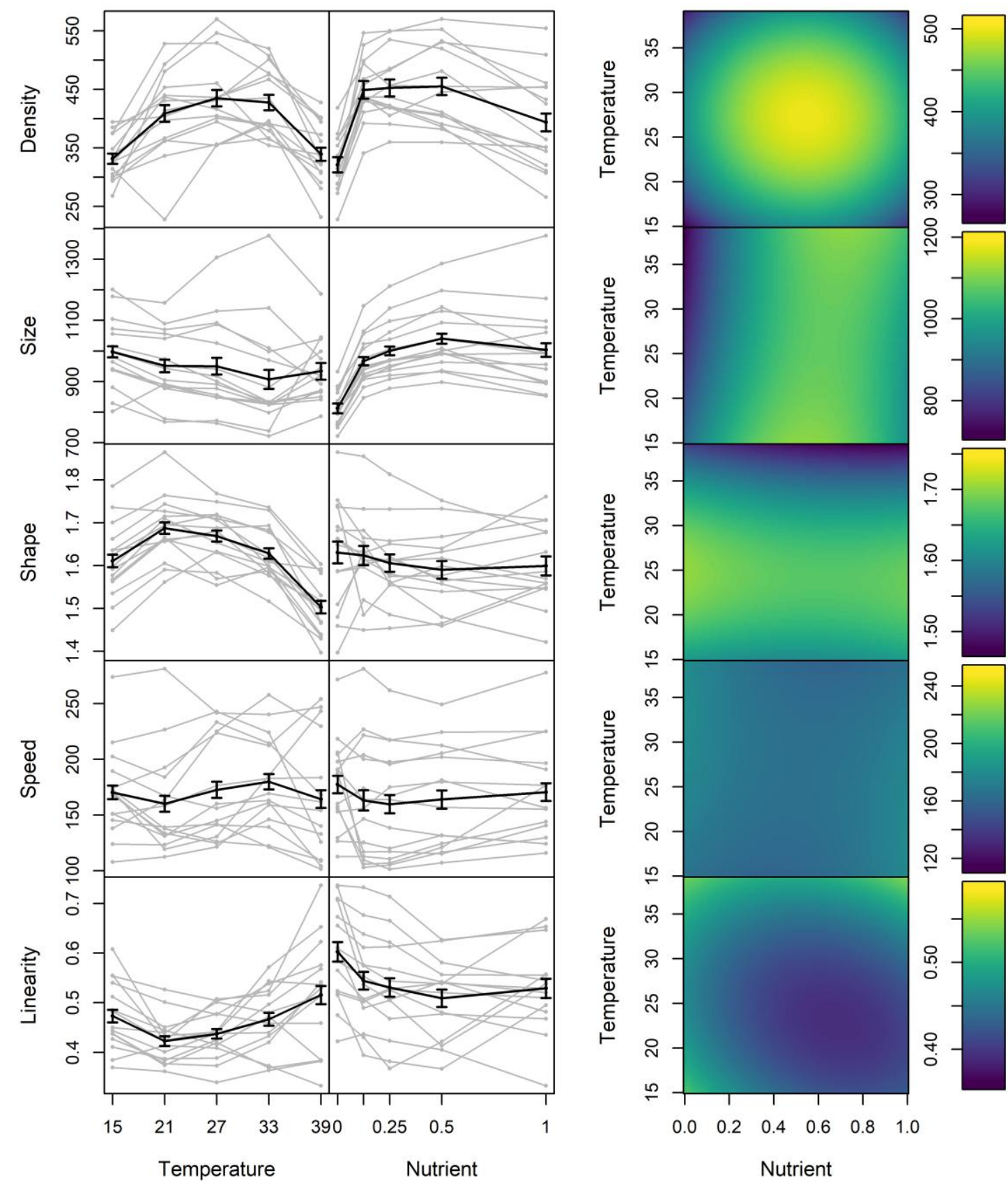

472 Figure 3: Reaction norms of the five traits according to the two environmental gradients

473 (temperature and nutrient concentration). (Left) Observed values for each genotype (grey lines,

474 averaged over 3 replicates) and averaged over all genotypes (black line \pm 2 standard errors) for each

475 environmental gradient separately. (Right) Predicted mean reaction norms across the $\mathrm{T} \times \mathrm{N}$ environmental space from the random regression mixed model. 


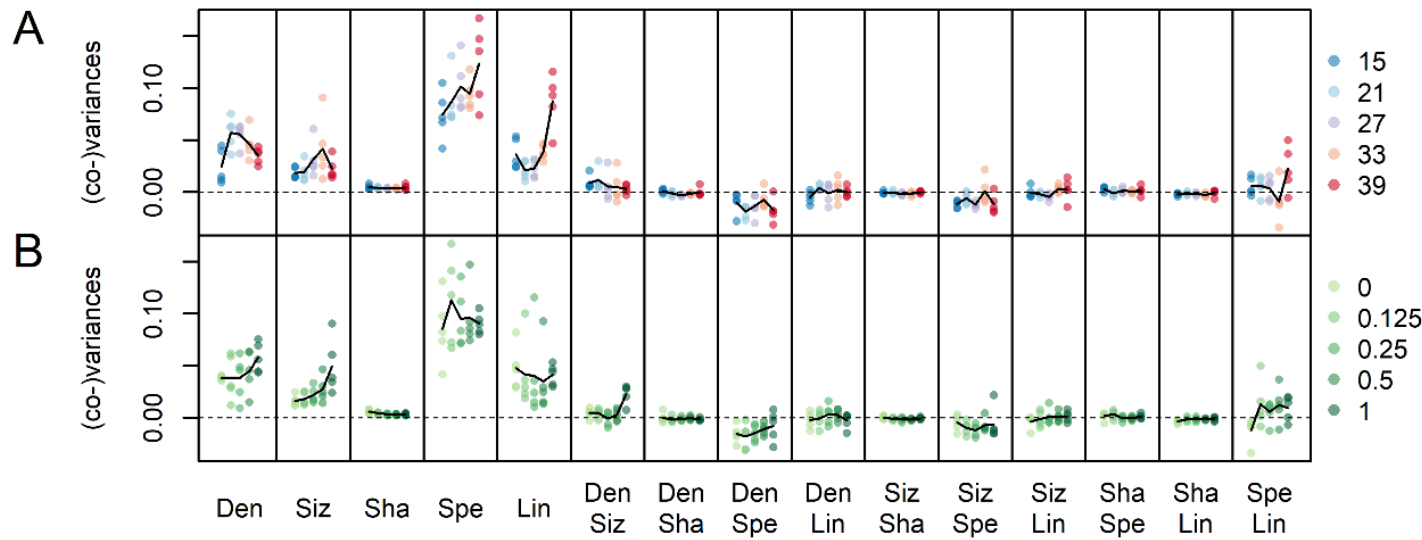

C

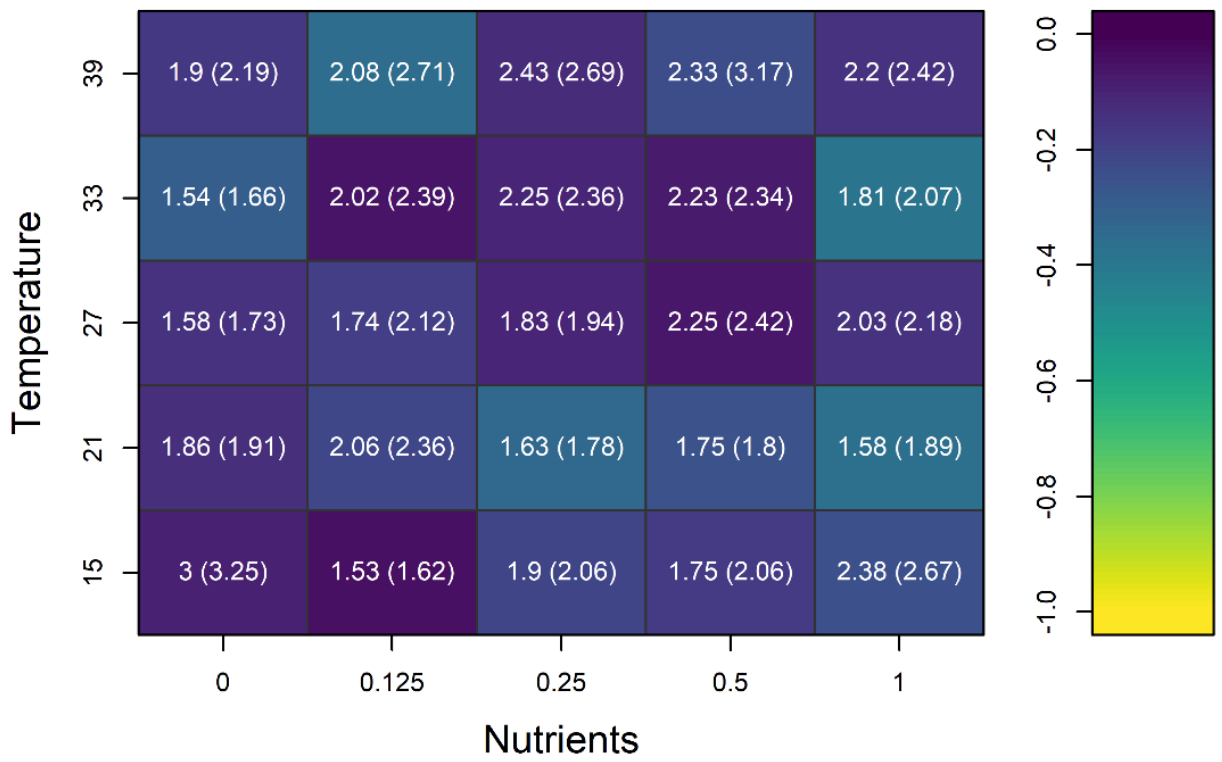

Nutrients

478 Figure 4: Values of variances and co-variances of the five phenotypic traits (Den: density, Siz: size,

479 Sha: shape, Spe: speed and Lin: linearity) for increasing values of temperatures $(\mathbf{A})$ and for increasing

480 values of nutrient concentration (B), with the average values (solid line). (C) Effective number of

481 dimensions of the G-matrices $\left(n_{D}\right)$ for the 25 environmental combinations, with the effective number

482 of dimensions for the same G-matrices with covariances forced at 0 ( $n_{D}{ }^{\prime}$ in parentheses) as the

483 maximum dimensionality adjusted for heterogenous variance in the five traits. The colours

484 correspond to the $\left(\left(n_{D}-1\right)-\left(n_{D}^{\prime}-1\right)\right) /\left(n_{D}^{\prime}-1\right)$ index, expressing whether dimensionality reduction is

485 entirely due to heterogeneous variances (index $=0$ ) or to strong covariances (index=-1). 

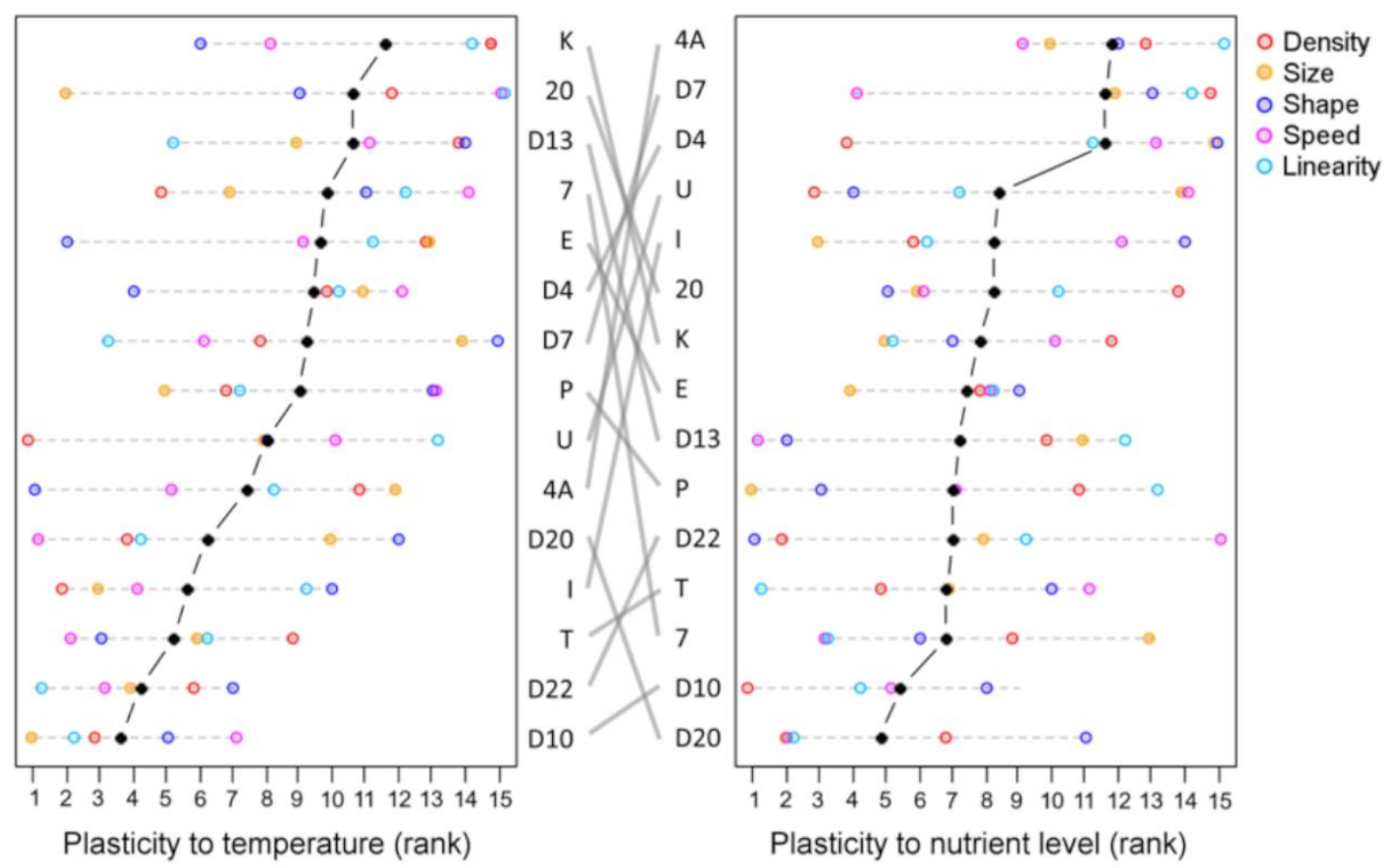

489 Figure 5: Plasticity magnitude rank of the genotypes (coefficient of variation of each trait across T 490 and across N) for plasticity to temperature (left) and to nutrient concentration (right). The genotypes 491 are sorted vertically according to their mean rank (black) across the 5 traits for each environmental factor. The rank positions of the genotypes in each gradient are connected in by grey lines to compare average plasticity of genotypes across the two environmental gradients 


\section{References}

Agrawal, A. A. (2001). Phenotypic plasticity in the interactions and evolution of species. Science, 294(5541), 321-326.

Aguirre, J., Hine, E., McGuigan, K., \& Blows, M. (2014). Comparing G: multivariate analysis of genetic variation in multiple populations. Heredity, 112(1), 21.

Altermatt, F., Fronhofer, E. A., Garnier, A., Giometto, A., Hammes, F., Klecka, J., ... Petchey, O. (2015). Big answers from small worlds: a user's guide for protist microcosms as a model system in ecology and evolution. Methods in Ecology and Evolution, 6(2), 218-231.

Auge, G. A., Leverett, L. D., Edwards, B. R., \& Donohue, K. (2017). Adjusting phenotypes via within-and across-generational plasticity. New Phytologist, 216(2), 343-349.

Bruno, J. F., \& Edmunds, P. J. (1997). Clonal variation for phenotypic plasticity in the coral Madracis mirabilis. Ecology, 78(7), 2177-2190.

Burghardt, K. T. (2016). Nutrient supply alters goldenrod's induced response to herbivory. Functional Ecology, 30(11), 1769-1778.

Callahan, H. S., Dhanoolal, N., \& Ungerer, M. C. (2005). Plasticity genes and plasticity costs: a new approach using an Arabidopsis recombinant inbred population. New Phytologist, 166(1), $129-140$.

Carroll, S. P., Hendry, A. P., Reznick, D. N., \& Fox, C. W. (2007). Evolution on ecological time-scales. Functional Ecology, 21(3), 387-393.

Chaine, A. S., Schtickzelle, N., Polard, T., Huet, M., \& Clobert, J. (2010). Kin-based recognition and social aggregation in a ciliate. Evolution: International Journal of Organic Evolution, 64(5), $1290-1300$.

Charmantier, A., McCleery, R. H., Cole, L. R., Perrins, C., Kruuk, L. E., \& Sheldon, B. C. (2008). Adaptive phenotypic plasticity in response to climate change in a wild bird population. Science, 320(5877), 800-803.

Chin, A. R., \& Sillett, S. C. (2016). Phenotypic plasticity of leaves enhances water-stress tolerance and 

807.

523 Delahaie, B., Charmantier, A., Chantepie, S., Garant, D., Porlier, M., \& Teplitsky, C. (2017). Conserved G-matrices of morphological and life-history traits among continental and island blue tit populations. Heredity, 119(2), 76.

Denver, R. J., Mirhadi, N., \& Phillips, M. (1998). Adaptive plasticity in amphibian metamorphosis: Response of Scaphiopus Hammondiitadpoles to habitat desiccation. Ecology, 79(6), 18591872.

DeWitt, T. J., \& Scheiner, S. M. (2004). Phenotypic plasticity: functional and conceptual approaches. Oxford University Press.

Dochtermann, N. A., \& Dingemanse, N. J. (2013). Behavioral syndromes as evolutionary constraints. Behavioral Ecology, 24(4), 806-811.

Doerder, F. P. (2019). Barcodes Reveal 48 New Species of Tetrahymena, Dexiostoma, and Glaucoma: Phylogeny, Ecology, and Biogeography of New and Established Species. Journal of Eukaryotic

Emlen, D. J., \& Nijhout, H. F. (2000). The development and evolution of exaggerated morphologies in insects. Annual Review of Entomology, 45(1), 661-708.

Fenster, C. B., Armbruster, W. S., Wilson, P., Dudash, M. R., \& Thomson, J. D. (2004). Pollination syndromes and floral specialization. Annu. Rev. Ecol. Evol. Syst., 35, 375-403.

Fjerdingstad, E. J., Schtickzelle, N., Manhes, P., Gutierrez, A., \& Clobert, J. (2007). Evolution of dispersal and life history strategies-Tetrahymena ciliates. BMC Evolutionary Biology, 7(1), 133.

Forsman, A. (2015). Rethinking phenotypic plasticity and its consequences for individuals, populations and species. Heredity, 115(4), 276.

Fritz, C. O., Morris, P. E., \& Richler, J. J. (2012). Effect size estimates: current use, calculations, and interpretation. Journal of Experimental Psychology: General, 141(1), 2. 
Fronhofer, E. A., Legrand, D., Altermatt, F., Ansart, A., Blanchet, S., Bonte, D., ... De Raedt, J. (2018). Bottom-up and top-down control of dispersal across major organismal groups. Nature Ecology \& Evolution, 2(12), 1859.

Geng, Y., van Klinken, R. D., Sosa, A., Li, B., Chen, J., \& Xu, C.-Y. (2016). The relative importance of genetic diversity and phenotypic plasticity in determining invasion success of a clonal weed in the USA and China. Frontiers in Plant Science, 7, 213.

Ghalambor, C. K., McKay, J. K., Carroll, S. P., \& Reznick, D. N. (2007). Adaptive versus non-adaptive phenotypic plasticity and the potential for contemporary adaptation in new environments. Functional Ecology, 21(3), 394-407.

Gienapp, P., Väisänen, R. A., \& Brommer, J. E. (2010). Latitudinal variation in breeding time reaction norms in a passerine bird. Journal of Animal Ecology, 79(4), 836-842.

Groot, M. P., Kooke, R., Knoben, N., Vergeer, P., Keurentjes, J. J., Ouborg, N. J., \& Verhoeven, K. J. (2016). Effects of multi-generational stress exposure and offspring environment on the expression and persistence of transgenerational effects in Arabidopsis thaliana. PloS One, 11(3), e0151566.

Hendry, A. P. (2015). Key questions on the role of phenotypic plasticity in eco-evolutionary dynamics. Journal of Heredity, 107(1), 25-41.

Hine, E., Chenoweth, S. F., Rundle, H. D., \& Blows, M. W. (2009). Characterizing the evolution of genetic variance using genetic covariance tensors. Philosophical Transactions of the Royal Society of London B: Biological Sciences, 364(1523), 1567-1578.

Hoverman, J. T., \& Relyea, R. A. (2007). How flexible is phenotypic plasticity? Developmental windows for trait induction and reversal. Ecology, 88(3), 693-705.

Husby, A., Nussey, D. H., Visser, M. E., Wilson, A. J., Sheldon, B. C., \& Kruuk, L. E. (2010). Contrasting patterns of phenotypic plasticity in reproductive traits in two great tit (Parus major) populations. Evolution: International Journal of Organic Evolution, 64(8), 2221-2237.

Jacob, S., Chaine, A. S., Huet, M., Clobert, J., \& Legrand, D. (2019). Variability in dispersal syndromes 
is a key driver of metapopulation dynamics in experimental microcosms. The American Naturalist, 194(5), 613-626.

575

576

577

578

579

580

581

582

583

584

585

586

587

588

589

590

591

592

593

594

595

596

597

598

Jacob, S., Clobert, J., Legrand, D., Schtickzelle, N., \& Chaine, A. S. (2016). Social information in cooperation and dispersal in Tetrahymena. In Biocommunication of Ciliates (pp. 235-252). Springer.

Jacob, S., Laurent, E., Haegeman, B., Bertrand, R., Prunier, J. G., Legrand, D., ... Clobert, J. (2018). Habitat choice meets thermal specialization: Competition with specialists may drive suboptimal habitat preferences in generalists. Proceedings of the National Academy of Sciences, 115(47), 11988-11993.

Jacob, S., Laurent, E., Morel-Journel, T., \& Schtickzelle, N. (2019). Fragmentation and the context-dependence of dispersal syndromes: matrix harshness modifies resident-disperser phenotypic differences in microcosms. Oikos.

Jacob, S., Legrand, D., Chaine, A. S., Bonte, D., Schtickzelle, N., Huet, M., \& Clobert, J. (2017). Gene flow favours local adaptation under habitat choice in ciliate microcosms. Nature Ecology \& Evolution, 1(9), 1407.

Kingsolver, J. G., Diamond, S. E., Siepielski, A. M., \& Carlson, S. M. (2012). Synthetic analyses of phenotypic selection in natural populations: lessons, limitations and future directions. Evolutionary Ecology, 26(5), 1101-1118.

Laughlin, D. C., \& Messier, J. (2015). Fitness of multidimensional phenotypes in dynamic adaptive landscapes. Trends in Ecology \& Evolution, 30(8), 487-496.

Ledón-Rettig, C. C., Richards, C. L., \& Martin, L. B. (2012). Epigenetics for behavioral ecologists. Behavioral Ecology, 24(2), 311-324.

Legrand, D., Larranaga, N., Bertrand, R., Ducatez, S., Calvez, O., Stevens, V. M., \& Baguette, M. (2016). Evolution of a butterfly dispersal syndrome. Proc. R. Soc. B, 283(1839), 20161533.

Miner, B. G., Sultan, S. E., Morgan, S. G., Padilla, D. K., \& Relyea, R. A. (2005). Ecological consequences of phenotypic plasticity. Trends in Ecology \& Evolution, 20(12), 685-692. 
Murren, C. J., Auld, J. R., Callahan, H., Ghalambor, C. K., Handelsman, C. A., Heskel, M. A., ... Maughan, H. (2015). Constraints on the evolution of phenotypic plasticity: limits and costs of phenotype and plasticity. Heredity, 115(4), 293.

Němcová, Y., Neustupa, J., Kvíderová, J., \& Řezáčová-Škaloudová, M. (2010). Morphological plasticity of silica scales of Synura echinulata (Synurophyceae) in crossed gradients of light and temperature-a geometric morphometric approach. Nova Hedwigia Beiheft, 136, 21-32.

Palacio-López, K., Beckage, B., Scheiner, S., \& Molofsky, J. (2015). The ubiquity of phenotypic plasticity in plants: a synthesis. Ecology and Evolution, 5(16), 3389-3400.

Pennekamp, F., Mitchell, K. A., Chaine, A., \& Schtickzelle, N. (2014). Dispersal propensity in Tetrahymena thermophila ciliates-a reaction norm perspective. Evolution, 68(8), 23192330.

Pennekamp, F., \& Schtickzelle, N. (2013). Implementing image analysis in laboratory-based experimental systems for ecology and evolution: a hands-on guide. Methods in Ecology and Evolution, 4(5), 483-492.

Pennekamp, F., Schtickzelle, N., \& Petchey, O. L. (2015). BEMOVI, software for extracting behavior and morphology from videos, illustrated with analyses of microbes. Ecology and Evolution, 5(13), 2584-2595.

Pfennig, D. W., Wund, M. A., Snell-Rood, E. C., Cruickshank, T., Schlichting, C. D., \& Moczek, A. P. (2010). Phenotypic plasticity's impacts on diversification and speciation. Trends in Ecology \& Evolution, 25(8), 459-467.

Pigliucci, M. (2001). Phenotypic plasticity: beyond nature and nurture. JHU Press.

Pigliucci, M., Murren, C. J., \& Schlichting, C. D. (2006). Phenotypic plasticity and evolution by genetic assimilation. Journal of Experimental Biology, 209(12), 2362-2367.

Poorter, H., Niinemets, Ü., Poorter, L., Wright, I. J., \& Villar, R. (2009). Causes and consequences of variation in leaf mass per area (LMA): a meta-analysis. New Phytologist, 182(3), 565-588.

Price, T. D., Qvarnström, A., \& Irwin, D. E. (2003). The role of phenotypic plasticity in driving genetic 

$1433-1440$.

R Core team. (2018). R: A language and environment for statistical computing. Vienna, Austria: $\mathrm{R}$ Foundation for Statistical Computing.

Réale, D., Garant, D., Humphries, M. M., Bergeron, P., Careau, V., \& Montiglio, P.-O. (2010). Personality and the emergence of the pace-of-life syndrome concept at the population level. Philosophical Transactions of the Royal Society B: Biological Sciences, 365(1560), 4051-4063.

Reed, T. E., Waples, R. S., Schindler, D. E., Hard, J. J., \& Kinnison, M. T. (2010). Phenotypic plasticity and population viability: the importance of environmental predictability. Proceedings of the Royal Society B: Biological Sciences, 277(1699), 3391-3400.

Relyea, R. A. (2004). Fine-tuned phenotypes: tadpole plasticity under 16 combinations of predators and competitors. Ecology, 85(1), 172-179.

Richards, C. L., Bossdorf, O., Muth, N. Z., Gurevitch, J., \& Pigliucci, M. (2006). Jack of all trades, master of some? On the role of phenotypic plasticity in plant invasions. Ecology Letters, 9(8), 981993.

Ris, N., Allemand, R., Fouillet, P., \& Fleury, F. (2004). The joint effect of temperature and host species induce complex genotype-by-environment interactions in the larval parasitoid of Drosophila, Leptopilina heterotoma (Hymenoptera: Figitidae). Oikos, 106(3), 451-456.

Roff, D. A. (2002). Life history evolution. Sunderland, USA: Sinauer Associates.

Sack, L., Melcher, P. J., Liu, W. H., Middleton, E., \& Pardee, T. (2006). How strong is intracanopy leaf plasticity in temperate deciduous trees? American Journal of Botany, 93(6), 829-839.

Schtickzelle, N., Fjerdingstad, E. J., Chaine, A., \& Clobert, J. (2009). Cooperative social clusters are not destroyed by dispersal in a ciliate. BMC Evolutionary Biology, 9(1), 251.

Sih, A., Bell, A., \& Johnson, J. C. (2004). Behavioral syndromes: an ecological and evolutionary overview. Trends in Ecology \& Evolution, 19(7), 372-378.

Smith, G., \& Ritchie, M. G. (2013). How might epigenetics contribute to ecological speciation? Current 
Zoology, 59(5), 686-696.

Stillwell, R. C., Wallin, W. G., Hitchcock, L. J., \& Fox, C. W. (2007). Phenotypic plasticity in a complex world: interactive effects of food and temperature on fitness components of a seed beetle. Oecologia, 153(2), 309-321.

Van Kleunen, M., \& Fischer, M. (2005). Constraints on the evolution of adaptive phenotypic plasticity in plants. New Phytologist, 166(1), 49-60.

van Snick Gray, E., \& Stauffer, J. (2004). Phenotypic plasticity: its role in trophic radiation and explosive speciation in cichlids (Teleostei: Cichlidae). Animal Biology, 54(2), 137-158.

Weider, L. J., \& Pijanowska, J. (1993). Plasticity of Daphnia life histories in response to chemical cues from predators. Oikos, 385-392.

West-Eberhard, M. J. (2003). Developmental plasticity and evolution. Oxford University Press.

Westneat, D. F., Hatch, M. I., Wetzel, D. P., \& Ensminger, A. L. (2011). Individual variation in parental care reaction norms: integration of personality and plasticity. The American Naturalist, $178(5), 652-667$.

Westneat, D. F., Potts, L. J., Sasser, K. L., \& Shaffer, J. D. (2019). Causes and consequences of phenotypic plasticity in complex environments. Trends in Ecology \& Evolution.

Winandy, L., Cote, J., Gesu, L. D., Pellerin, F., Trochet, A., \& Legrand, D. (2019). Local predation risk and matrix permeability interact to shape movement strategy. Oikos.

Wright, I. J., Reich, P. B., Cornelissen, J. H., Falster, D. S., Groom, P. K., Hikosaka, K., ... Oleksyn, J. (2005). Modulation of leaf economic traits and trait relationships by climate. Global Ecology and Biogeography, 14(5), 411-421.

Yeh, P. J., \& Price, T. D. (2004). Adaptive phenotypic plasticity and the successful colonization of a novel environment. The American Naturalist, 164(4), 531-542.

Zera, A. J., \& Denno, R. F. (1997). Physiology and ecology of dispersal polymorphism in insects. Annual Review of Entomology, 42(1), 207-230.

Zufall, R. A., Dimond, K. L., \& Doerder, F. P. (2013). Restricted distribution and limited gene flow in 
the model ciliate T etrahymena thermophila. Molecular Ecology, 22(4), 1081-1091. 